\title{
Survival pathway of cholangiocarcinoma via AKT/mTOR signaling to escape RAF/MEK/ERK pathway inhibition by sorafenib
}

\author{
KENTA YOKOI $^{1}$, AKIRA KOBAYASHI ${ }^{1}$, HIROAKI MOTOYAMA ${ }^{1}$, MASATO KITAZAWA ${ }^{1}$, \\ AKIRA SHIMIZU $^{1}$, TSUYOSHI NOTAKE ${ }^{1}$, TAKAHIDE YOKOYAMA ${ }^{1}$, \\ TOMIO MATSUMURA ${ }^{2}$, MICHIKO TAKEOKA ${ }^{1}$ and SHIN-ICHI MIYAGAWA ${ }^{1}$ \\ ${ }^{1}$ Department of Surgery, Shinshu University School of Medicine; ${ }^{2}$ Department of Molecular Oncology, \\ Shinshu University Graduate School of Medicine, Matsumoto 390-8621, Japan
}

Received July 12, 2017; Accepted December 7, 2017

DOI: $10.3892 /$ or.2017.6153

\begin{abstract}
Cholangiocarcinoma (CCC) is a strongly aggressive malignancy for which surgical resection is the only potential curative therapy. Sorafenib, a multikinase inhibitor of the RAF/MEK/ERK pathway, is a molecular-targeted drug that is approved for hepatocellular carcinoma (HCC) but not for CCC. The differences in signaling pathway characteristics under sorafenib treatment between HCC (HLF, Huh7, PLC/PRF/5) and CCC (RBE, YSCCC, Huh28) cell lines were therefore investigated using cell proliferation, western blotting, and apoptosis analyses. Sorafenib inhibited cell growth significantly less in CCC cells than in HCC cells, with lower suppression of ERK phosphorylation. Significantly decreased AKT Ser473 phosphorylation in HCC cells, and conversely enhanced phosphorylation of AKT Ser473 and mTORC2 in CCC cells, were observed with sorafenib treatment. Disassembly of the mTORC2 complex in RBE cells with siRNA targeting Rictor resulted in the downregulation of AKT Ser473 phosphorylation and enhanced apoptosis presumably via increased FOXO1, which consequently suppressed RBE cell proliferation. Phosphorylation of mTORC1 and autophagy were not influenced by sorafenib in CCC cells. Simultaneous administration of everolimus to suppress activated mTORC1 in RBE cells revealed that combined everolimus and sorafenib treatment under mTORC2 disassembly could enhance growth inhibition through the suppression of both sorafenib- and everolimusdependent AKT Ser473 phosphorylation in addition to the
\end{abstract}

Correspondence to: Dr Akira Kobayashi, Department of Surgery, Shinshu University School of Medicine, 3-1-1 Asahi, Matsumoto 390-8621, Japan

E-mail: kbys@shinshu-u.ac.jp

Key words: sorafenib, cholangiocarcinoma, AKT Ser473, mTORC2 disassembly, everolimus inhibition of mTORC1 phosphorylation. Prevention of escape by AKT/mTOR signaling from the RAF/MEK/ERK pathway in sorafenib treatment by suppressing mTORC2 activity may lead to promising new approaches in CCC therapy.

\section{Introduction}

Originating in the epithelial cells lining the biliary tree, cholangiocarcinoma (CCC) is an aggressive malignancy with a poor prognosis. CCC represents $10-15 \%$ of total hepatobiliary tumors (1) and its incidence and mortality are continuously increasing worldwide (2). Although the 5-year survival rate of patients receiving curative resection for CCC is $30-40 \%$, individuals with unresectable CCC generally survive less than 12 months after diagnosis (3). Thus, the establishment of effective clinical molecular markers for early diagnosis and targeted molecular therapies are urgently needed for CCC.

Among the numerous inhibitors of tyrosine kinase, sorafenib (BAY 43-9006) has attracted considerable attention. Sorafenib inhibited Raf-1 (4) and BRAF (5), both members of the RAF/mitogen-activated protein kinase (MEK)/extracellular signal-related kinase (ERK) signaling pathway, and suppressed the proliferation and growth of several human tumor cell lines and xenograft models. The drug also exhibited significant activity against multiple receptor tyrosine kinases involved in tumor progression, including vascular endothelial growth factor receptor (VEGFR)-2, VEGFR-3, c-KIT, Flt-3, and platelet-derived growth factor receptor $\beta$ (5).

Sorafenib is effective for hepatocellular carcinoma (HCC) by virtue of prolonged median survival in advanced-stage patients (6). The effects of sorafenib, however, are far less understood for CCC. Sorafenib exerted low activity in a phase II CCC trial (7), and not even a combination of sorafenib and erlotinib could exhibit clinical activity in patients with biliary cancers in a phase II trial (8). Thus, sorafenib is the first molecular-targeted therapy to be approved for HCC, but not for CCC, despite the fact that the liver and bile duct are derived from the same embryological origin.

The in vitro antitumor activity of sorafenib in human CCC has been assessed in several signaling pathways. Blockage 
of the MAPK pathway by sorafenib inhibited cell proliferation through cell cycle arrest (9), and sorafenib accelerated STAT3 dephosphorylation and induced TRAIL-mediated apoptosis (10).

Recently, the phosphoinositide 3-kinase (PI3K)/AKT/ mammalian target of rapamycin (mTOR) pathway was implicated in sorafenib-resistant HCC, whereby constitutive activation of the mTOR pathway was present in drug-resistant HCC cells (11). Increased AKT phosphorylation was also witnessed in established sorafenib-resistant HCC cells (12). We therefore focused our attention on the AKT/mTOR pathway as a CCC escape mechanism from $\mathrm{RAF} / \mathrm{MEK} / \mathrm{ERK}$-mediated cell death under sorafenib based on comparisons of HCC and CCC.

Two forms of mTOR protein complexes exist. mTORC1, defined by the Raptor scaffolding protein (13), is sensitive to rapamycin. Activation of $\mathrm{mTORC} 1$ triggers mitochondrial oxidative metabolism and lipogenesis, which are critically important in tumorigenesis (14). mTORC1 is also a negative regulator of autophagy (15). Characterized by Rictor, mTORC2 phosphorylates AKT on Ser 473, regulates forkhead box protein (FOXO) activation, and modifies the actin cytoskeleton with oaxilin and Rho GTPases (16). Inhibition of mTORC2 by Rictor disruption decreases AKT-dependent tumor progression in lapatinib-resistant HER2-amplified breast cancers (17). Accordingly, we examined the influence of sorafenib on the AKT/mTOR pathway to observe how dissociation of the mTORC2 component by Rictor knockdown altered this pathway in CCC. Since activated mTORC1 has been reported in CCC, we also combined everolimus with sorafenib to simultaneously suppress mTORC1 under mTORC2 disassembly.

\section{Materials and methods}

Cell lines and culture. The human HCC cell lines HLF, Huh7, and PLC/PRF/5 and human intrahepatic CCC cell line Huh28 were obtained from the Japanese Collection of Research Bioresources (JCRB) Cell Bank (Osaka, Japan). The human intrahepatic CCC cell lines RBE and YSCCC were procured from the RIKEN Cell Bank (Ibaraki, Japan). HCC cells were maintained in Dulbecco's modified Eagle's medium and CCC cells were maintained in RPMI-1640. All cultures were supplemented with $10 \%$ fetal bovine serum (FBS), $100 \mathrm{U} / \mathrm{ml}$ penicillin, and $100 \mu \mathrm{g} / \mathrm{ml}$ streptomycin in a humidified atmosphere of $5 \% \mathrm{CO}_{2}$ at $37^{\circ} \mathrm{C}$.

Analysis of cell proliferation and anchorage-independent growth assay. The MTT assay was used for analyzing cell proliferation. At $24 \mathrm{~h}$ after inoculation of $\mathrm{HCC}$ and CCC cells, DMSO $(0.1 \%$ in culture medium) or 5 or $10 \mu \mathrm{M}$ sorafenib were administered and the cells were cultured for an additional $24 \mathrm{~h}$. Ten microliters WST-1 reagent (Roche Diagnostics $\mathrm{GmbH}$, Mannheim, Germany) was added to each well and the absorbance was assessed at $450 \mathrm{~nm}$ after $1 \mathrm{~h}$ of incubation using an Epoch microplate reader (BioTek Instruments, Inc., Winooski, VT, USA). We performed anchorage-independent assays according to a previously described method (18). Briefly, cells were mixed in $0.36 \%$ agar-containing medium with $10 \%$ FBS containing DMSO or 5 or $10 \mu \mathrm{M}$ sorafenib. The mixture was placed on a bed of $0.72 \%$ agar-containing medium with $10 \%$ FBS and DMSO or 5 or $10 \mu \mathrm{M}$ sorafenib in 35-mm dishes. Three weeks after the inoculation, the colony areas were assessed using NIH ImageJ software (Rockville, MD, USA).

Apoptosis assay. One day after inoculation, RBE cells were treated with DMSO or 5 or $10 \mu \mathrm{M}$ sorafenib for $24 \mathrm{~h}$ and cellular apoptosis was examined by Annexin $\mathrm{V}$ and propidium iodide (PI) staining using an Annexin V-FITC Apoptosis kit (BioVision, Inc., Milpitas, CA, USA) according to the manufacturer's protocol. Following staining, the cells were analyzed using flow cytometry (FACS) on a FACSCalibur device (BD Biosciences, Franklin Lakes, NJ, USA).

Western blot analysis. Cells were lysed in lysis buffer containing $50 \mathrm{mM}$ Tris- $\mathrm{HCl} \mathrm{pH} 7.4,150 \mathrm{mM} \mathrm{NaCl}, 1 \%$ Triton $\mathrm{X}-100,1 \%$ sodium deoxycholate, $0.1 \%$ SDS, 1X EDTA-free proteinase inhibitor cocktail and $1 \mathrm{X}$ phosphatase inhibitor cocktail (both from Roche Diagnostics $\mathrm{GmbH}$ ) for $15 \mathrm{~min}$ at $4^{\circ} \mathrm{C}$. The lysates were separated by $15 \%$ SDS-PAGE and the proteins were transferred onto nitrocellulose membranes. After blocking with $5 \%$ skim milk for $1 \mathrm{~h}$, the membranes were incubated with primary antibodies overnight at $4^{\circ} \mathrm{C}$, and then secondary antibodies conjugated with horseradish peroxidase were applied for $1 \mathrm{~h}$. Immune complexes were developed with ECL Select $^{\mathrm{TM}}$ Western blotting detection reagent (Amersham, GE Healthcare Life Sciences, Chicago, IL USA). The results were photographed with a Molecular Imager ChemiDoc XRS device (Bio-Rad Laboratories, Inc., Hercules, CA, USA). The integrated density of the immunoblots was analyzed by Image Lab Software (Bio-Rad Laboratories, Inc.) and results were expressed as a percentage of the immunoblots of the internal control, $\beta$-actin. The anti-human antibodies used were as follows: rabbit monoclonal antibodies: ERK (cat. no. 4695), p-ERK (cat. no. 4376), AKT (cat. no. 4691), 0020P-AKT Thr 308 (cat. no. 2965), p-AKT Ser473 (cat. no. 4060), p-mTOR s2448 (cat. no. 5536), Rictor (cat. no. 9476) and FOXO1 (cat. no. 2880) (all from Cell Signaling Technology, Inc., Danvers, MA, USA); rabbit polyclonal antibodies: p-mTOR s2481 (cat. no. 2974), p-FOXO1/3 (cat. no. 9464) (Cell Signaling Technology, Inc.), LC3 (cat. no. PM036; MBL, Nagoya, Japan); and mouse monoclonal antibody: $\beta$-actin (cat. no. A5441; Sigma-Aldrich, St. Louis, MO, USA). The anti- $\beta$-actin antibody was used at a 1:3,000 dilution and the other antibodies were used at a 1:1,000 dilution.

Knockdown of Rictor via siRNA transfection for disassembly of the mTORC2 complex in RBE cells. Silencer Select siRNA was purchased from Life Technologies/Thermo Fisher Scientific (Carlsbad, CA, USA) and modified to target human Rictor by reverse transfection (19). Either $10 \mu \mathrm{M}$ Silencer Select non-targeting negative control or $10 \mu \mathrm{M}$ Rictor siRNA was mixed with Lipofectamine RNAiMAX (Life Technologies/ Thermo Fisher Scientific) according to the manufacturer's instructions and added to $35-\mathrm{mm}$ tissue culture plates. The cells were then plated onto siRNA/Lipofectamine RNAiMAX complexes at a density of $1 \times 10^{5}$ cells/well in RPMI-1640 containing $5 \mathrm{mM}$ glucose and 10\% FBS. At $48 \mathrm{~h}$ after transfection, Rictor knockdown was confirmed by western blotting and the cells were subjected to ensuing experiments. 

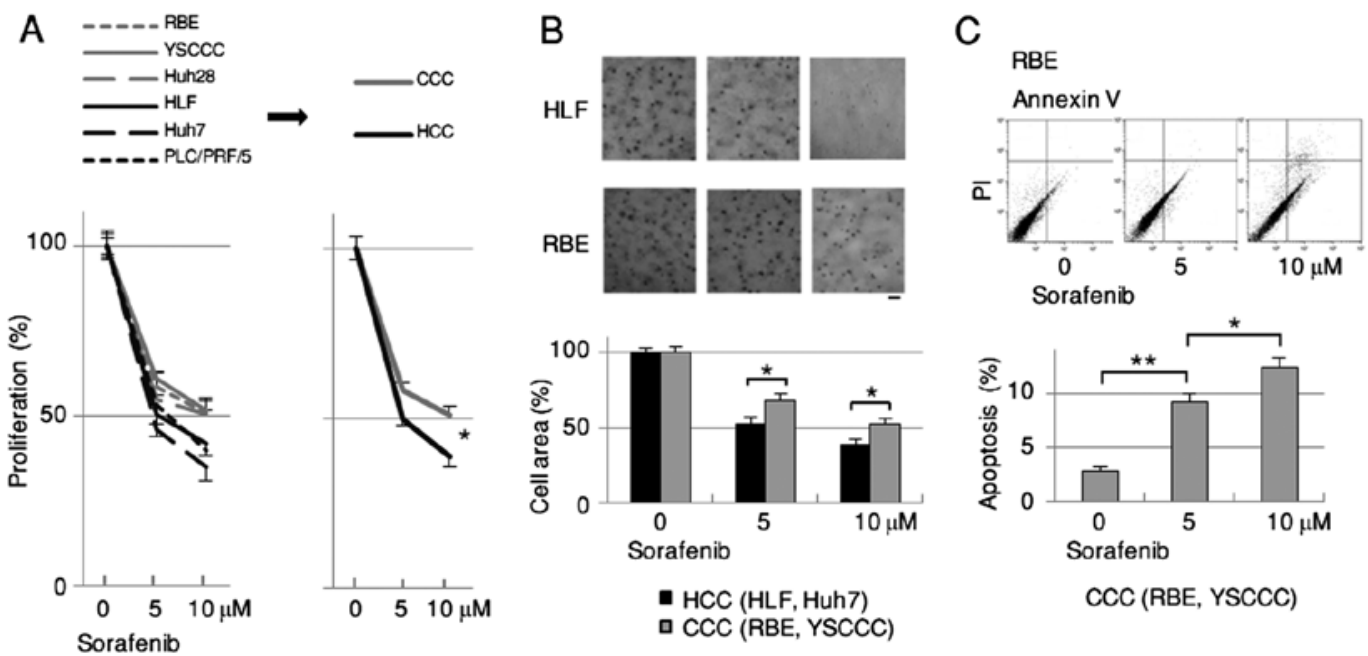

Figure 1. Effect of sorafenib on growth inhibition is significantly less in CCC than in HCC. (A) Comparison of the effect of sorafenib on growth inhibition of HCC (HLF, Huh7 and PLC/PRF/5) and CCC (RBE, YSCCC and Huh28) cells as detected by the MTT assay ( $\mathrm{n}=6$ ). Data are given as percentages of the viability of cells. Percentage values were combined for each cell type (right). (B) Comparison of the effect of sorafenib on growth inhibition of HCC (HLF, Huh7) and CCC (RBE, YSCCC) cells as examined by the anchorage-independent soft agar assay $(\mathrm{n}=10)$. (C) Apoptosis of RBE and YSCCC cells treated with sorafenib as assessed by FACS $(\mathrm{n}=6) .{ }^{*} \mathrm{P}<0.05,{ }^{* *} \mathrm{P}<0.01$.

A

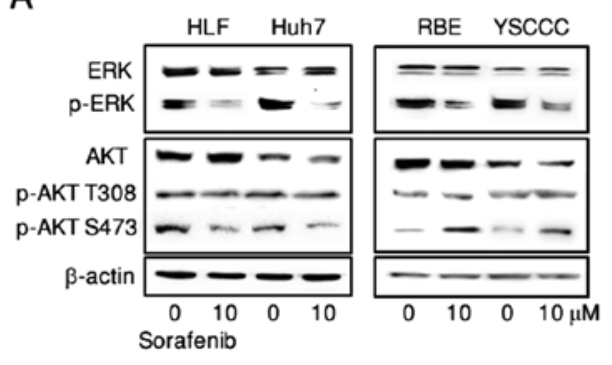

B

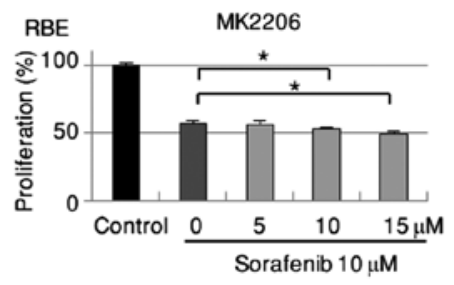

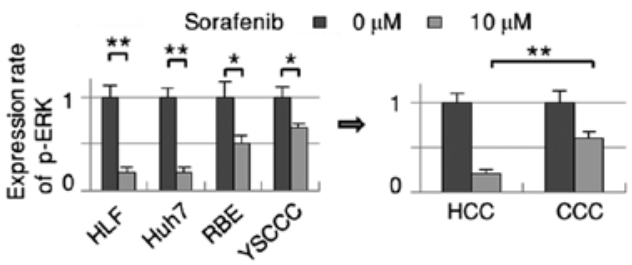

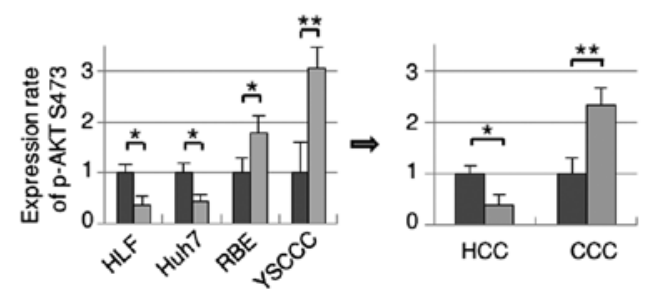

Figure 2. Phosphorylation of AKT Ser473 is decreased in HCC but increased in CCC by sorafenib. (A) Phosphorylation of ERK and AKT was compared by western blot assaying between HCC (HLF and Huh7) and CCC (RBE and YSCCC) cells treated with sorafenib (n=4). Data are presented as the rate of the integrated density of immunoblots. (B) Effects of the AKT inhibitor MK2206 on the proliferation of RBE cells treated with sorafenib as determined by the MTT assay $(n=6) .{ }^{*} \mathrm{P}<0.05,{ }^{* *} \mathrm{P}<0.01$.

Statistical analysis. Statistical significance was evaluated using the Student's t-test on the data of 3-6 experiments for each assay. $\mathrm{P}<0.05$ was accepted as statistically significant. All values were expressed as the mean \pm standard error of the mean.

\section{Results}

Sorafenib inhibits growth significantly less in CCC than in $H C C$. Since sorafenib is the first molecular-targeted drug approved for HCC but not for CCC, we compared its effects on the proliferation of $\mathrm{HCC}$ and CCC cells. The degree of growth inhibition by sorafenib was significantly less in CCC (RBE,
YSCCC, Huh28) than in HCC (HLF, Huh7, PLC/PRF/5) cells as assessed by MTT assay (Fig. 1A). These results were supported by the anchorage-independent assay comparing HCC (HLF, Huh7) and CCC (RBE, YSCCC) cells 3 weeks after cell plating (Fig. 1B). The apoptosis assay using FACS revealed that the population of Annexin V-positive and PI-negative cells increased dose-dependently by sorafenib in RBE and YSCCC cells (Fig. 1C).

Phosphorylation of AKT Ser473 is decreased in HCC but increased in CCC by sorafenib. Based on sorafenib's known inhibitory effects of sorafenib on the RAF/MEK/ ERK signaling pathway, we examined the drug's impact on 
A

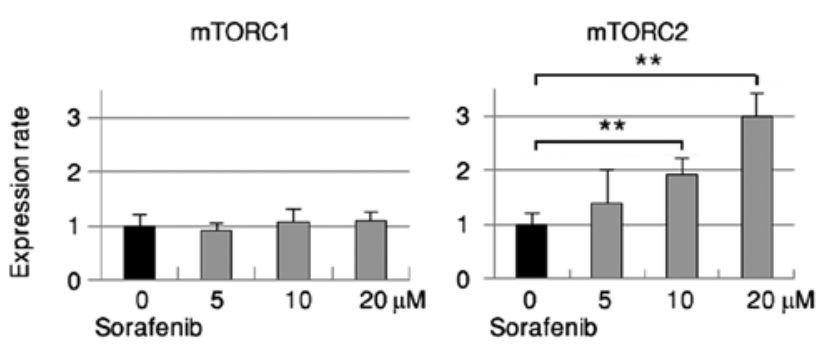

B
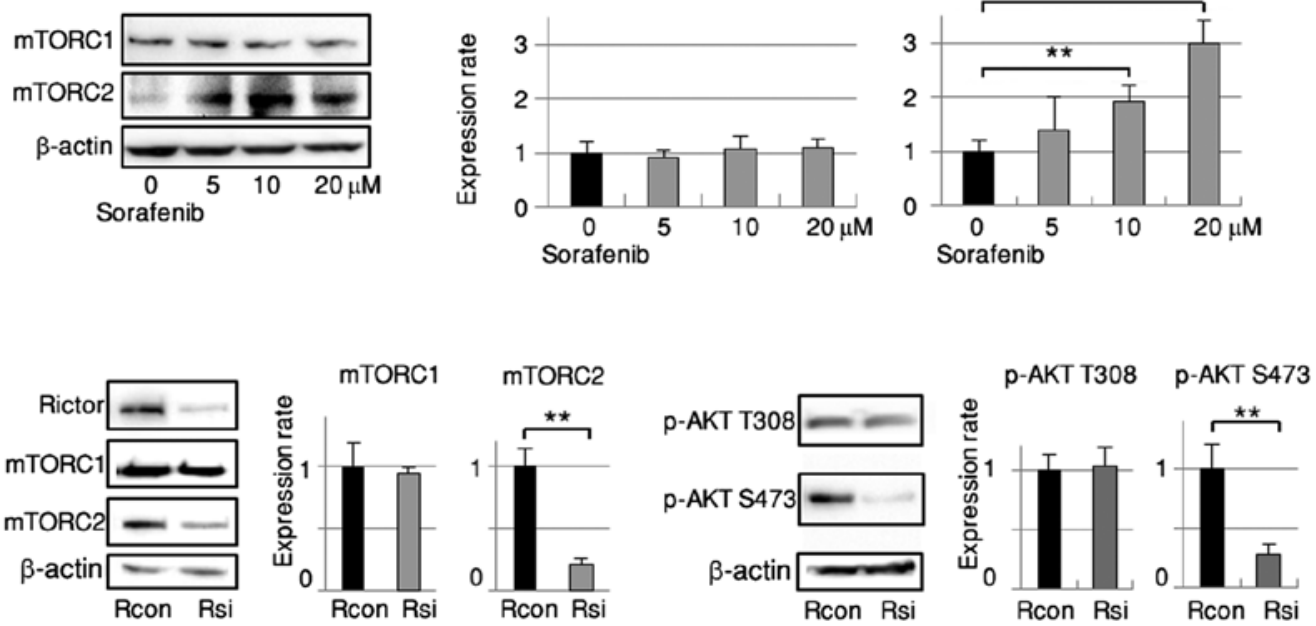

Figure 3. Sorafenib upregulates mTORC2 phosphorylation, and then mTORC2 is dissociated using siRNA targeting Rictor in RBE cells. (A) Phosphorylation of mTORC1 and mTORC2 in RBE cells treated with sorafenib (n=5). (B) Confirmation of Rictor knockdown and the consequent phosphorylation levels of mTORC1, mTORC2, AKT Thr308 and Ser473 $(\mathrm{n}=4)$. Rcon, control siRNA; Rsi, siRNA targeting Rictor. ${ }^{* *} \mathrm{P}<0.01$.

this signal transduction pathway in $\mathrm{HCC}$ and $\mathrm{CCC}$ cells. Administration of sorafenib markedly suppressed ERK phosphorylation in both cell types, with a significantly lower suppression in CCC cells (Fig. 2A). Regarding AKT Ser473, a signaling molecule in the PI3 kinase pathway, phosphorylation was significantly decreased in HCC cells by sorafenib treatment but significantly increased in CCC cells (Fig. 2A). We observed no marked alterations in AKT Thr308 phosphorylation in either cell type. These findings raised the biochemical possibility of an escape mechanism from the major RAF/MEK/ERK signaling pathway elicited by activation of the AKT/mTOR signaling cascade in sorafenib treatment for CCC.

We next inhibited the sorafenib-dependent increase of AKT Ser473 phosphorylation in RBE cells using the selective allosteric AKT inhibitor MK2206 to clarify the drug's growth inhibitory effect. High dose $(10$ and $15 \mu \mathrm{M})$ administration of MK2206 for $72 \mathrm{~h}$ significantly enhanced the suppression of cell growth caused by $10 \mu \mathrm{M}$ sorafenib-treated RBE cells in the MTT assay (Fig. 2B).

Downregulation of AKT Ser473 phosphorylation is obtained via disassembly of the mTORC2 complex induced by Rictor silencing in RBE cells. Twenty-four hours after treatment with sorafenib, dose-dependent activation of mTORC2 (mTOR Ser2481 phosphorylation) was detected by western blot analysis (Fig. 3A) with no apparent alteration in mTORC1 activation (mTOR Ser 2448 phosphorylation) (20). Since mTORC2 is located upstream of AKT Ser473, we silenced it by means of siRNA to abrogate AKT Ser473 phosphorylation. RBE cells were transfected with control siRNA or siRNA targeting Rictor, an essential and specific component of mTORC2. As shown in Fig. 3B, Rictor expression was markedly decreased and the phosphorylation of mTORC2 was significantly reduced. Phosphorylation of AKT Ser473 was significantly suppressed as well. Rictor knockdown did not affect mTORC1 or AKT Thr308 phosphorylation.
Disassembly of mTORC2 prevents sorafenib-dependent activation of the AKT/mTOR pathway and enhances the antitumor efficacy of sorafenib in RBE cells without affecting autophagy. The increase of AKT Ser473 phosphorylation by sorafenib was significantly abrogated in mTORC2-disassembled RBE cells as detected by western blotting (Fig. 4A), while mTORC2 disassembly did not affect the phosphorylation of AKT Thr308. In cell growth assays, RBE cell proliferation was dose-dependently suppressed by sorafenib treatment. This suppression was significantly stronger in mTORC2-disassembled cells than in controls (Fig. 4B).

The growth suppression induced by sorafenib treatment under mTORC2 disassembly corresponded with an increase in apoptosis (Fig. 4C) as detected by FACS. Therefore, we examined the effect of sorafenib on FOXO1 as an inducer of cell death. Sorafenib treatment elicited marked upregulation of FOXO1/3 phosphorylation along with a reduction of FOXO1 expression (Fig. 4D). Collectively, sorafenib appeared to enhance mTORC2 and AKT Ser473 phosphorylation and decrease FOXO1, which may have suppressed apoptosis and consequently facilitated cell survival. Since mTORC1 is a negative regulator of autophagy, we searched for alterations in mTORC1 and autophagy by sorafenib. Disassembly of mTORC2 with sorafenib did not alter mTORC1 phosphorylation in RBE cells (Fig. 4E). Sorafenib did not affect autophagy in CCC cells as determined by western blot analysis of LC3-II/total LC3 expression (Fig. 4F). Thus, sorafenib played no apparent role in the autophagy-related pathway of CCC cells.

Schematic representation of an escape mechanism via AKT/ mTOR signaling from the RAF/MEK/ERK pathway evoked by sorafenib in RBE cells. Based on the aforementioned findings, we speculated that the AKT/mTOR pathway activated by sorafenib represented an escape mechanism (Fig. 5, right) from the RAF/MEK/ERK signaling pathway by which sorafenib normally exerted its cell-death properties (Fig. 5, left). In the RAF/MEK/ERK pathway, inhibited ERK 
A

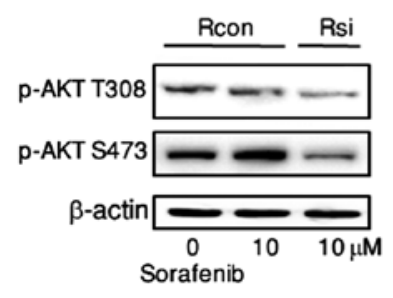

C

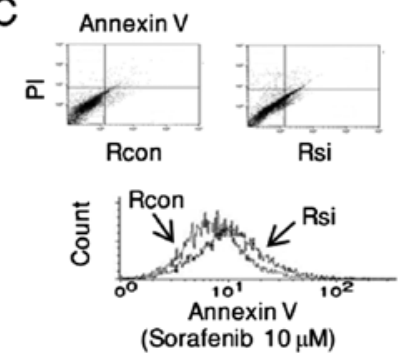

E

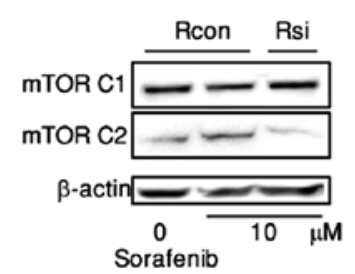

B
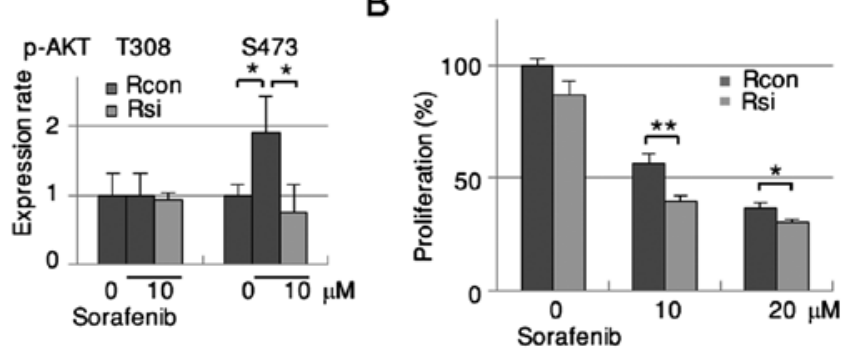

D
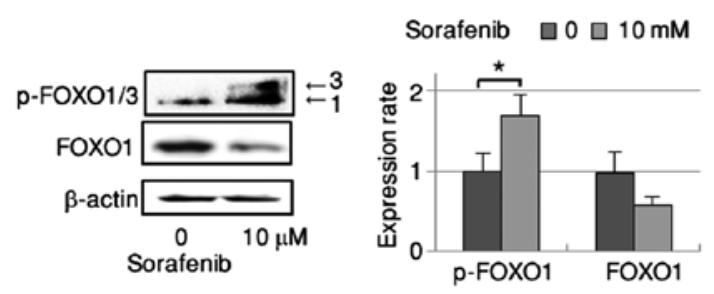

F
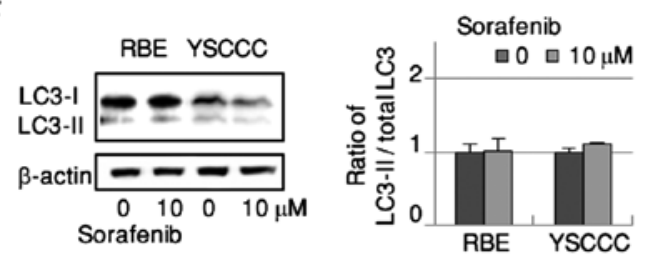

Figure 4. Sorafenib exhibits a growth inhibitory effect under disassembly of mTORC2 in RBE cells without affecting mTORC1 or autophagy. Effects of mTORC2 disassembly with sorafenib treatment on (A) AKT Thr308 and Ser473 phosphorylation $(n=4)$, $(B)$ cell proliferation $(n=6)$, and $(C)$ apoptosis. Representative data of triplicate experiments. (D) Effect of sorafenib on FOXO1/3 phosphorylation and FOXO1 expression as detected by western blotting $(n=3)$. (E) Effect of mTORC2 disassembly on mTORC1 phosphorylation. (F) Effect of sorafenib on autophagy as evaluated by the expression of LC3I and LC3II by western blot analysis in CCC (RBE and YSCCC) cells $(n=6)$. Rcon, control siRNA; Rsi, siRNA targeting Rictor. "P<0.05, ${ }^{* *} \mathrm{P}<0.01$.

phosphorylation and cell proliferation by sorafenib were lower in CCC cells than in HCC cells. In the AKT/mTOR escape pathway, sorafenib upregulated the phosphorylation of AKT Ser473 via mTORC2 activation without influencing mTORC1 or AKT Thr308 phosphorylation through a yet unknown initial receptor. The upregulated AKT Ser473 by sorafenib decreased the expression level of FOXO1, presumably leading to a decrease in apoptosis and consequently facilitating cell survival. As disassembled mTORC2 with sorafenib did not influence the phosphorylation of $\mathrm{mTORC}$, the cascade from AKT Ser473 to mTORC1 by sorafenib was unaffected, and thus sorafenib did not alter autophagy in CCC cells.

Combination of everolimus with sorafenib under mTORC2 disassembly enhances the inhibitory effects on cell growth in RBE cells. Everolimus is a potent inhibitor of mTORC1. Disassembly of mTORC2 suppressed the everolimus-dependent phosphorylation of mTORC2 and AKT Ser473 (Fig. 6A). Everolimus was then combined with sorafenib under mTORC2 disassembly in RBE cells. As shown in Fig. 6B, everolimus or sorafenib alone suppressed cell growth, with the latter being enhanced by mTORC2 dissociation. Furthermore, under mTORC2 disassembly, combined treatment with everolimus and sorafenib more strongly suppressed cell growth than did sorafenib alone. This enhanced growth suppression corresponded with evident downregulation of mTORC1, mTORC2,

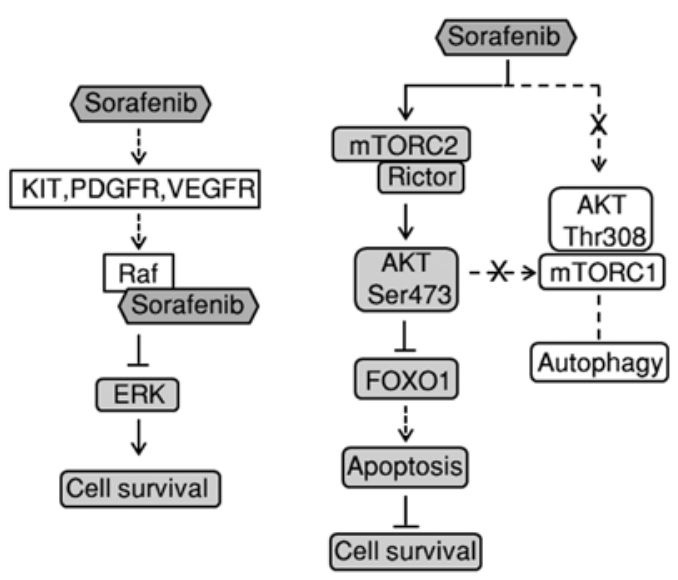

< Major pathway > < Escape pathway >

Figure 5. Schematic representation of the escape mechanism via AKT/mTOR signaling from the RAF/MEK/ERK pathway evoked by sorafenib in RBE cells. In the RAF/MEK/ERK major signaling pathway, ERK phosphorylation was suppressed by sorafenib. In the AKT/mTOR escape pathway, sorafenib activated mTORC2 and AKT Ser473 and inhibited apoptosis via suppression of FOXO1, which consequently increased cell growth independently of autophagy.

and AKT Ser473 phosphorylation (Fig. 6C). Unexpectedly, the phosphorylation of AKT Ser473 was strongly suppressed 
A

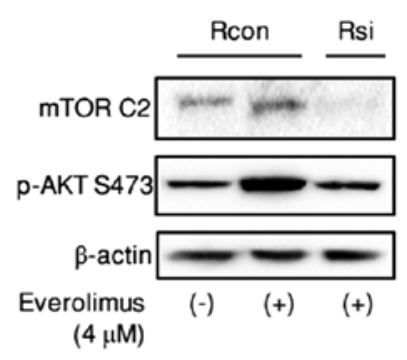

B

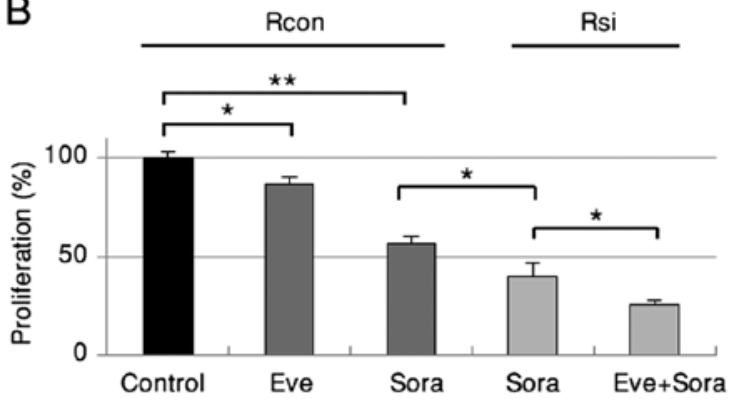

(Sorafenib: $10 \mu \mathrm{M}$, Everolimus: $4 \mu \mathrm{M}$ )
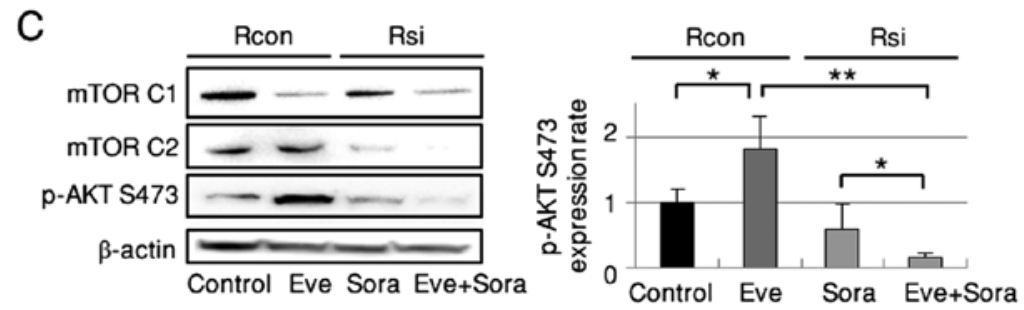

(Sorafenib: $10 \mu \mathrm{M}$, Everolimus: $4 \mu \mathrm{M}$ )

Figure 6. Combined everolimus and sorafenib treatment under disassembly of mTORC2 more effectively inhibits proliferation of RBE cells. (A) Effect of mTORC2 disassembly on everolimus-dependent AKT Ser473 phosphorylation. Representative data of triplicate experiments. (B) Inhibitory effect on cell proliferation of everolimus alone, sorafenib alone, sorafenib under mTORC2 disassembly, and combined everolimus and sorafenib under mTORC2 disassembly $(\mathrm{n}=6)$. (C) Phosphorylation of mTORC1, mTORC2, and AKT Ser473 by everolimus alone, sorafenib under mTORC2 disassembly, and combined everolimus and sorafenib under mTORC2 disassembly $(\mathrm{n}=5)$. Rcon, control siRNA; Rsi, siRNA targeting Rictor; Sora, sorafenib; Eve, everolimus. "P<0.05, ${ }^{* *} \mathrm{P}<0.01$.

by combined everolimus/sorafenib treatment under mTORC2 disassembly.

\section{Discussion}

The present investigation uncovered a possible escape mechanism of CCC from the RAF/MEK/ERK pathway by AKT/mTOR signaling during sorafenib treatment. Since disassembly of the mTORC2 complex led to an inhibition of AKT Ser473 phosphorylation and suppressed cell growth, the prevention of AKT/mTOR pathway function by suppressing mTORC2 during sorafenib treatment may be a promising therapeutic option for CCC.

Constitutive activation of the AKT/mTOR pathway was recently reported in sorafenib-resistant HCC cells (11). Decreases in AKT Ser473 phosphorylation in sorafenibsensitive HCC cells vs. increases in sorafenib-resistant HCC cells with sorafenib treatment have been documented as well (21). In the present study, the increased phosphorylation of AKT Ser473 in CCC by sorafenib was similar to that in sorafenib-resistant HCC cells. The inhibitory effects of the drug on the RAF/MEK/ERK signaling pathway (9) and STAT3 pathway (10) in CCC are well known. However, the AKT/mTOR pathway has not yet been addressed, and thus we focused on this cascade as a possible escape mechanism from cell death via the RAF/MEK/ERK signaling pathway in sorafenib treatment for CCC and searched for ways to abrogate the increase in AKT Ser473 phosphorylation.

Our initial attempts to prevent sorafenib-dependent AKT Ser473 phosphorylation employed the AKT inhibitor MK2206, which could inhibit endogenous (22) and everolimus-elicited (23) phosphorylation of AKT in CCC. Similarly,
MK2206 administration with sorafenib significantly enhanced the suppression of cell growth at high concentrations and prolonged treatment. We therefore searched for a more effective method than MK2206 to suppress the sorafenib-dependent increase of AKT Ser473 phosphorylation.

Sorafenib administration to RBE cells significantly increased mTORC2 without altering mTORC1. Therefore, we disassembled the mTORC2 complex using siRNA that targeted Rictor (19) to effectively suppress the phosphorylation of AKT Ser473 in RBE cells. mTORC2 regulates the phosphorylation of AKT Ser473 (13). Our results demonstrated that sorafenib administration following siRNA treatment significantly reduced the phosphorylation of AKT Ser473 and more strongly suppressed cell proliferation as compared with sorafenib treatment alone in RBE cells. This finding was consistent with a study revealing that the depletion of Rictor decreased AKT Ser473 phosphorylation and tumor cell survival in multiple amplified human breast cancer cell lines, including those with acquired resistance to lapatinib (17).

Since mTORC2 disassembly increased apoptosis, we examined the involvement of the transcription factor FOXO1 in cell-death activity. Sorafenib upregulated FOXO1/3 phosphorylation and downregulated FOXO1 in RBE cells. According to Salazar et al, increased phosphorylation of AKT on Ser473 enhanced the phosphorylation and inactivation of FOXO3 (24). Phosphorylated FOXO exits the nucleus for degradation. Moreover, mTORC2 inhibition increased the expression level of FOXO1/3, and knockdown of FOXO3 abrogated rhein-induced apoptosis (25). Thus, we considered that the suppression of sorafenib-dependent AKT Ser473 phosphorylation by mTORC2 disassembly induced apoptosis via increased FOXO1. 
Autophagy is a double-edged sword that depends on its cellular context. Sorafenib treatment led to autophagy in HCC cells, while pharmacological inhibition of this autophagy increased apoptosis and decreased cell viability (26). In addition, the drug activated AKT in sorafenib-resistant HCC cells, and inhibition of this activation reversed the acquired resistance to sorafenib by switching autophagy from cell survival to cell death (27). In CCC cells, however, the negative autophagy regulator mTORC1 was not affected by sorafenib or mTORC2 disassembly. Sorafenib did not influence autophagy in CCC as evidenced by LC3-II, although autophagy was increased in HCC (data not shown). This indicated the existence of an escape mechanism from cell death with sorafenib in CCC that was unrelated to autophagy.

Our body of findings enabled the elucidation of a possible escape mechanism via the AKT/mTOR pathway from the major RAF/MEK/ERK pathway under sorafenib treatment in an RBE cell line (Fig. 5). Sorafenib activated mTORC2 and AKT Ser473 and inhibited apoptosis via suppressed FOXO1, which consequently increased cell growth independently of autophagy.

Lastly, since we detected constitutively phosphorylated mTORC1 in RBE cells, we attempted to suppress mTORC1 by everolimus. The combined administration of sorafenib and everolimus under mTORC2 disassembly produced additional growth inhibitory effects by abrogating both sorafenib- and everolimus-dependent AKT Ser473 phosphorylation in RBE cells.

In HCC, the efficacy of combined therapy with rapamycin analogs and sorafenib has been demonstrated by the suppression of mTORC1 activation and cell growth in sorafenib-less-sensitive lines (27). Moreover, increased AKT phosphorylation observed not only with sorafenib, but also with rapamycin, in sorafenib-resistant HCC cells implied that feedback activation of AKT may limit the rapamycin-mediated antitumor effects (17). mTORC2 has been proposed to be rapamycin insensitive (28). However, a recent study described that everolimus induced mTORC2mediated AKT Ser473 activation in ovarian carcinoma and that inhibition of mTORC2 during treatment enhanced the antitumor effects (29). Moreover, Pignochino et al demonstrated that everolimus increased mTORC2 activity with mTORC1 suppression, and the combination of sorafenib and everolimus potentiated the antiproliferative effect of each drug with decreased phosphorylation of mTORC2 and AKT Ser473 in osteosarcoma (30). Therefore, we hypothesized that a feedback increase of AKT Ser473 phosphorylation elicited by both sorafenib and everolimus would be simultaneously blocked by disassembly of the mTORC 2 component in RBE cells. In our experiments, disassembly of mTORC 2 suppressed everolimus-dependent AKT Ser473 activation, and the combination of everolimus and sorafenib under mTORC2 disassembly significantly prevented AKT Ser473 phosphorylation and synergistically exerted a regulatory effect on RBE cell proliferation. Thus, dissociation of mTORC2 may disable the escape mechanism from sorafenib and permit the mTORC1 inhibitory effect of everolimus without a feedback increase of AKT. We examined the phosphorylation of mTORC2 and AKT treated with a combination of everolimus and sorafenib. Both phosphorylations were slightly reduced compared with those produced by everolimus alone (data not shown), although the degree was smaller than a study on osteosarcoma by Pignochino et al (30). As they reported, this combination may dissociate mTORC2 to some degree and contribute to the markedly suppressed phosphorylation of AKT Ser473 by combination treatment under mTORC2 disassembly in RBE cells shown in Fig. 6C.

In conclusion, although RAF kinases are one of the main molecules targeted by sorafenib, intervention of active AKT/mTOR signaling in the RAF/MEK/ERK pathway may be one of the mechanisms responsible for the resistance of CCC to sorafenib. AKT/mTOR pathway regulation is exceedingly complex due to multiple feedback loops and direct activation mechanisms. Nonetheless, suppression of mTORC2 activity by microRNA targeting of Rictor should be considered in potential therapies combining sorafenib and everolimus for CCC malignancies.

\section{Acknowledgements}

Sorafenib was kindly provided by Bayer Health Care Pharmaceuticals (Berlin, Germany). This study was supported by a grant-in-aid for General Scientific Research (JP26293300) from the Ministry of Education, Culture, Sports, Science and Technology of Japan.

\section{References}

1. Lazaridis $\mathrm{KN}$ and Gores GJ: Cholangiocarcinoma. Gastroenterology 128: 1655-1667, 2005.

2. Patel T: Worldwide trends in mortality from biliary tract malignancies. BMC Cancer 2: 10, 2002.

3. Gores GJ: Cholangiocarcinoma: Current concepts and insights. Hepatology 37: 961-969, 2003.

4. Lyons JF, Wilhelm S, Hibner B and Bollag G: Discovery of a novel Raf kinase inhibitor. Endocr Relat Cancer 8: 219-225, 2001.

5. Wilhelm SM, Carter C, Tang L, Wilkie D, McNabola A, Rong H, Chen C, Zhang X, Vincent P, McHugh M, et al: BAY 43-9006 exhibits broad spectrum oral antitumor activity and targets the RAF/MEK/ERK pathway and receptor tyrosine kinases involved in tumor progression and angiogenesis. Cancer Res 64: 7099-7109, 2004

6. Llovet JM, Ricci S, Mazzaferro V, Hilgard P, Gane E, Blanc JF, de Oliveira AC, Santoro A, Raoul JL, Forner A, et al; SHARP Investigators Study Group: Sorafenib in advanced hepatocellular carcinoma. N Engl J Med 359: 378-390, 2008.

7. Bengala C, Bertolini F, Malavasi N, Boni C, Aitini E, Dealis C, Zironi S, Depenni R, Fontana A, Del Giovane C, et al: Sorafenib in patients with advanced biliary tract carcinoma: A phase II trial. Br J Cancer 102: 68-72, 2010

8. El-Khoueiry AB, Rankin C, Siegel AB, Iqbal S, Gong IY, Micetich KC, Kayaleh OR, Lenz HJ and Blanke CD: S0941: A phase 2 SWOG study of sorafenib and erlotinib in patients with advanced gallbladder carcinoma or cholangiocarcinoma. Br J Cancer 110: 882-887, 2014.

9. Sugiyama H, Onuki K, Ishige K, Baba N, Ueda T, Matsuda S, Takeuchi K, Onodera M, Nakanuma Y, Yamato M, et al: Potent in vitro and in vivo antitumor activity of sorafenib against human intrahepatic cholangiocarcinoma cells. J Gastroenterol 46: 779-789, 2011.

10. Blechacz BR, Smoot RL, Bronk SF, Werneburg NW, Sirica AE and Gores GJ: Sorafenib inhibits signal transducer and activator of transcription-3 signaling in cholangiocarcinoma cells by activating the phosphatase shatterproof 2. Hepatology 50: 1861-1870, 2009.

11. Masuda M, Chen WY, Miyanaga A, Nakamura Y, Kawasaki K, Sakuma T, Ono M, Chen CL, Honda $\mathrm{K}$ and Yamada T: Alternative mammalian target of rapamycin (mTOR) signal activation in sorafenib-resistant hepatocellular carcinoma cells revealed by array-based pathway profiling. Mol Cell Proteomics 13: $1429-1438,2014$ 
12. Zhai B, Hu F, Jiang X, Xu J, Zhao D, Liu B, Pan S, Dong X, Tan G, Wei Z, et al: Inhibition of Akt reverses the acquired resistance to sorafenib by switching protective autophagy to autophagic cell death in hepatocellular carcinoma. Mol Cancer Ther 13: 1589-1598, 2014.

13. Chiarini F, Evangelisti C, McCubrey JA and Martelli AM: Current treatment strategies for inhibiting mTOR in cancer. Trends Pharmacol Sci 36: 124-135, 2015.

14. Laplante $M$ and Sabatini DM: mTOR signaling at a glance. J Cell Sci 122: 3589-3594, 2009.

15. Dunlop EA and Tee AR: mTOR and autophagy: A dynamic relationship governed by nutrients and energy. Semin Cell Dev Biol 36: 121-129, 2014.

16. Laplante M and Sabatini DM: mTOR signaling in growth control and disease. Cell 149: 274-293, 2012.

17. Morrison Joly M, Hicks DJ, Jones B, Sanchez V, Estrada MV, Young C, Williams M, Rexer BN, Sarbassov D, Muller WJ, et al: Rictor/mTORC2 drives progression and therapeutic resistance of HER2-amplified breast cancers. Cancer Res 76: 4752-4764, 2016.

18. Horiuchi A, Nikaido T, Taniguchi S and Fujii S: Possible role of calponin $\mathrm{h} 1$ as a tumor suppressor in human uterine leiomyosarcoma. J Natl Cancer Inst 91: 790-796, 1999.

19. Soares HP, Ming M, Mellon M, Young SH, Han L, Sinnet-Smith J and Rozengurt E: Dual PI3K/mTOR Inhibitors Induce Rapid Overactivation of the MEK/ERK Pathway in Human Pancreatic Cancer Cells through Suppression of mTORC2. Mol Cancer Ther 14: 1014-1023, 2015.

20. Copp J, Manning G and Hunter T: TORC-specific phosphorylation of mammalian target of rapamycin (mTOR): phospho-Ser2481 is a marker for intact mTOR signaling complex 2. Cancer Res 69: $1821-1827,2009$.

21. Guan DX, Shi J, Zhang Y, Zhao JS, Long LY, Chen TW, Zhang EB, Feng YY, Bao WD, Deng YZ, et al: Sorafenib enriches epithelial cell adhesion molecule-positive tumor initiating cells and exacerbates a subtype of hepatocellular carcinoma through TSC2-AKT cascade. Hepatology 62: 1791-1803, 2015.

22. Wilson JM, Kunnimalaiyaan S, Kunnimalaiyaan M and Gamblin TC: Inhibition of the AKT pathway in cholangiocarcinoma by MK2206 reduces cellular viability via induction of apoptosis. Cancer Cell Int 15: 13, 2015.
23. Ewald F, Grabinski N, Grottke A, Windhorst S, Nörz D, Carstensen L, Staufer K, Hofmann BT, Diehl F, David K, et al: Combined targeting of AKT and mTOR using MK-2206 and RAD001 is synergistic in the treatment of cholangiocarcinoma. Int J Cancer 133: 2065-2076, 2013.

24. Salazar M, Lorente M, García-Taboada E, Pérez Gómez E, Dávila D, Zúñiga-García P, María Flores J, Rodríguez A, Hegedus Z, Mosén-Ansorena D, et al: Loss of Tribbles pseudokinase-3 promotes Akt-driven tumorigenesis via FOXO inactivation. Cell Death Differ 22: 131-144, 2015.

25. Wang J, Liu S, Yin Y, Li M, Wang B, Yang L and Jiang Y: FOXO3-mediated up-regulation of Bim contributes to rheininduced cancer cell apoptosis. Apoptosis 20: 399-409, 2015.

26. Shimizu S, Takehara T, Hikita H, Kodama T, Tsunematsu H, Miyagi T, Hosui A, Ishida H, Tatsumi T, Kanto T, et al: Inhibition of autophagy potentiates the antitumor effect of the multikinase inhibitor sorafenib in hepatocellular carcinoma. Int J Cancer 131: 548-557, 2012.

27. Huynh H, Ngo VC, Koong HN, Poon D, Choo SP, Thng CH, Chow P, Ong HS, Chung A and Soo KC: Sorafenib and rapamycin induce growth suppression in mouse models of hepatocellular carcinoma. J Cell Mol Med 13 (8B): 2673-2683, 2009.

28. Jacinto E, Loewith R, Schmidt A, Lin S, Rüegg MA, Hall A and Hall MN: Mammalian TOR complex 2 controls the actin cytoskeleton and is rapamycin insensitive. Nat Cell Biol 6: 1122-1128, 2004.

29. Hisamatsu T, Mabuchi S, Matsumoto Y, Kawano M, Sasano T, Takahashi R, Sawada K, Ito K, Kurachi H, Schilder RJ, et al: Potential role of mTORC2 as a therapeutic target in clear cell carcinoma of the ovary. Mol Cancer Ther 12: 1367-1377, 2013.

30. Pignochino Y, Dell'Aglio C, Basiricò M, Capozzi F, Soster M, Marchiò S, Bruno S, Gammaitoni L, Sangiolo D, Torchiaro E, et al: The combination of sorafenib and everolimus abrogates mTORC1 and mTORC2 upregulation in osteosarcoma preclinical models. Clin Cancer Res 19: 2117-2131, 2013. 